\title{
A better best system account of lawhood
}

\author{
Jonathan Cohen · Craig Callender
}

Published online: 1 April 2009

(C) The Author(s) 2009. This article is published with open access at Springerlink.com

\begin{abstract}
Perhaps the most significant contemporary theory of lawhood is the Best System (/MRL) view on which laws are true generalizations that best systematize knowledge. Our question in this paper will be how best to formulate a theory of this kind. We'll argue that an acceptable MRL should (i) avoid inter-system comparisons of simplicity, strength, and balance, (ii) make lawhood epistemically accessible, and (iii) allow for laws in the special sciences. Attention to these problems will bring into focus a useful menu of novel MRL theories, some of which solve problems the original MRL theory could not. Hence we conceive of the paper as moving toward a better Best System theory of laws.
\end{abstract}

Keywords Laws of nature · Mill-Ramsey-Lewis · Natural kinds . Explosive realism $\cdot$ Special sciences

The laws of Nature, that is to say the laws of God, plainly made every human being a law unto himself, we must steadfastly refuse to obey those laws, and we must as steadfastly stand by the conventions which ignore them, since the statutes furnish us peace, fairly good government, and stability, and therefore

This work is fully collaborative; the authors are listed anti-alphabetically.

J. Cohen $(\bowtie) \cdot$ C. Callender

Department of Philosophy, University of California, San Diego, 9500 Gilman Drive, La Jolla,

CA 92093-0119, USA

e-mail: joncohen@aardvark.ucsd.edu

C. Callender

e-mail: ccallender@ucsd.edu 
are better for us than the laws of God, which would soon plunge us into confusion and disorder and anarchy if we should adopt them.

Mark Twain, in Bernard DeVoto, ed., Mark Twain in Eruption: Hitherto Unpublished Pages About Men and Events (1940).

Are there laws of nature, and if so, what are they? Three answers to these two questions stand out in the philosophical literature. The first, the No-Laws answer, denies that an important part of the scientific enterprise is finding anything like laws of nature. There are symmetries, causes, and maybe general principles found inside very abstract idealized models, but nothing worthy of the title 'laws' (Cartwright 1983; van Fraassen 1989; Giere 1999). Absent motivation from science, there is no reason to believe laws exist. A second view, the Governing answer, insists that there are genuine laws of nature and furthermore that these laws govern or even produce the events of the world (Dretske 1977; Tooley 1977; Swoyer 1982; Armstrong 1983; Shoemaker 1998; Maudlin 2007). Just as librarians enforce the rules of book borrowing and policemen enforce traffic rules, so some Governing theorists think that necessitarian relations, primitive accessibility relations, or primitive universals enforce certain behaviors upon the events of the world. Other advocates of Governing are silent on how the laws manage these feats, but insist that they do and treat laws with the requisite governing powers as primitive. The third view, the NonGoverning conception, has it that there are genuine laws of nature, but that they do not govern or produce the events of the world. The mosaic of events displays certain patterns, and it is in the features of some of these patterns that we find laws.

Perhaps the most significant contemporary Non-Governing theory of lawhood is the so-called Best System (or MRL) view associated with Mill, Ramsey and Lewis. According to MRL, the laws of nature are the true generalizations that best systematize our scientific knowledge. MRL has a lot going for it, and we share the view that it represents the most promising current approach to lawhood-especially for empiricists, but for non-empiricists as well.

The MRL approach is superior to other non-Governing views: it is admirably realist when compared against projectivism (e.g., Goodman 1954; Ayer 1956; Ward 2002), and suffers from far fewer problems than the naïve regularity analysis (Swartz 1985). At the same time, it also seems to compare favorably to both NoLaws and Governing answers. From the perspective of MRL, the postulation of governing laws is akin to the postulation of an Unmoved Mover to explain motion. The worry is that, while governing laws explain why there are certain patterns in the mosaic of events, nothing explains why the particular laws, primitive universals, accessibility relations, (etc.) hold. Consequently, the laws become the Unenforced Enforcers of patterns. But if we have to stop explanation there, why not simply stick with the patterns of events? The Governing conception appears to foist metaphysical commitments on scientists merely by virtue of their appeal to laws. By contrast, MRL seems far less metaphysically freighted. It is grounded in nothing more than the properties, individuals, and events in the world, and formal (deductive) relations defined over statements about these entities, that are already recognized by our best scientific descriptions of the way the world is. The modesty of MRL's extra- 
scientific apparatus has made the view seem attractive to thinkers who are inclined to defer to the best scientific descriptions of the world-both to Humeans (and others who forswear necessary connections) and more generally to those who prefer fewer metaphysical posits to more. ${ }^{1}$

Of course, advocates of No Laws views believe that such deference will not reveal the presence of laws. There is a challenge here. Once we have rejected the Governing conception of laws, is there enough left to the concept of lawhood to find it playing an important role in the scientific enterprise? Or should laws be viewed as no more crucial to science than symmetries, explanatory strategies, and other important but not wholly central features of science?

Contrary to the No Laws view, we believe that it is very hard to make sense of actual scientific practice and the history of science without invoking laws of nature. MRL states that laws are the generalizations that result from a trade-off between the competing virtues of simplicity and informativeness. Scientists certainly see themselves as engaged in the project of finding such generalizations:

that's our quest: to look for a simple set of physical principles... from which everything we know about physics can be derived (Weinberg 1987, p. 64).

[I]t is possible to condense the enormous mass of results to a large extentthat is to find laws which summarize... (Feynman et al. 1963, p. 1-1).

Going beyond what they say, it is clear especially since Newton that scientists have sought general but simple principles applicable to systems with very general features. Virtually every science textbook contains frequent appeal to simple principles that cover a vast array of phenomena in the field. Even philosophers skeptical of laws recognize that scientific theorizing is a process of carefully balancing simplicity and strength (e.g., Cartwright 1983, p. 144). And in many cases the result of this process is a set of fundamental principles that are taken to describe the essence of a theory. The laws of mechanics, principle of natural selection, Malthusian principle, and law of supply and demand are all attempts to provide such principles. We stress that such attempts are not exclusive to physics. In population ecology, for example, Berryman (2003) argues that there are a set of five general principles that "are sufficient to describe, classify and explain all known patterns of population dynamics" (700; see also Colyvan 2003). Recognizing in science the attempt to produce small sets of basic principles as a result of balancing simplicity and informativeness is the central and powerful insight that motivates MRL (and also, we believe, the unificationist theory of explanation). We submit that it would be hard to make sense of the history of science without this crucial insight (whether we call these principles 'laws' or not). That said, the fact that MRL scores well on a list of features we want from laws-such as distinguishing laws from accidental true generalizations, countenancing vacuous laws, supporting counterfactuals, and connections to objective probabilities (Loewer 1996) — convinces us that MRL laws are worthy of the name.

\footnotetext{
1 Please note, however, that the doctrine of Humean supervenience-roughly, that everything supervenes upon local matters of particular fact-is logically distinct from MRL. Indeed, many versions of MRL we'll discuss below are at odds with Humean supervenience.
} 
We'd like to defend MRL if we can. However, the view faces some important problems - some of which have been previously noted, some not-and it is worth working through possible solutions to see if MRL can be saved. The problem that will be our point of departure in this paper concerns whether the view can avoid appeal to the inter-system comparisons of strength, simplicity, and balance that we believe are unavailable. ${ }^{2}$ While others have noted this problem, its seriousness has been underappreciated. For if this worry goes unanswered, MRL laws are not connected to scientific practice in the manner advertised, and so MRL does not enjoy the main advantage claimed for it over both Governing and No Law conceptions of laws. However, this is not the only constraint on MRL that we want to impose; we'll argue that, in addition, an adequate MRL must (i) make lawhood epistemically accessible through ordinary scientific inquiry, and (ii) allow for laws in the special sciences. Our question in this paper will be whether and how one can formulate a version of MRL that satisfies all of these desiderata. Attention to these problems has the additional value of bringing into focus a number of very different-looking variations of MRL that one might consider as responses. The result, we believe, is a useful menu of novel MRL theories, some of which solve problems the original MRL theory could not. Hence we conceive of the paper as moving toward a better Best System theory of laws.

Here's how we'll proceed. After rehearsing the MRL theory briefly, we'll motivate the desiderata of avoiding inter-system comparisons of simplicity, strength and balance, securing epistemic accessibility, and providing for special science laws (Sect. 1). We'll then consider a series of increasingly permissive ways of securing these benefits for MRL, and explore their costs and benefits (Sects. 2-4). Finally, we'll suggest what we think is the most attractive version of MRL that emerges from this discussion (Sect. 5).

\section{MRL laws and three desiderata}

The MRL view begins from a conception of our knowledge of the world as a deductive system containing axioms and results derived from those axioms. ${ }^{3}$ of course, there will be different ways of arranging our knowledge of the world into such a deductive system. Some such systems will be stronger than others in the sense that they contain (among their axioms and derived consequences) more truths about the world than others-some such systematizations carry more information about the world, or, equivalently, rule out more possible ways the world might have been, than others. Other true deductive systems will be simpler than others in the senses that they include a smaller set of independent axioms or are syntactically less complex. ${ }^{4}$ Significantly, strength and simplicity are competing virtues: adding more

\footnotetext{
${ }^{2}$ We believe that this is the most important objection against MRL. Much of the remaining criticism of MRL in the literature consists of various imagined counter-examples; but we take these worries to have been answered convincingly by Earman (1986) and Loewer (1996).

3 For canonical statements, see Lewis (1973, 73ff, 1983, pp. 365-368), Earman (1986); Lewis (1994a, 478ff) extends the view to incorporate objective chances.

${ }^{4}$ Understanding the simplicity of a given system is a difficult problem that we won't attempt to resolve here. For a non-syntactic alternative, it is possible to regard simplicity in a manner more connected to
} 
axioms to a system increases a system's strength at the cost of simplicity, while taking away axioms increases simplicity at the cost of strength. The MRL approach to the problem of lawhood is to say that a true generalization is a law if and only if it is an axiom of all the "Best Systems" - axiomatic systematizations that best balance strength and simplicity. For example, Einstein's field equation should plausibly count as a law on this account because any deductive system lacking it would be inferior in strength (by leaving out many truths about the world) or simplicity (by recapturing the otherwise left-out truths only by including a huge list of underived axioms about what objects attract what others). In contrast, the true generalization that all attendees in your metaphysics seminar are seated is plausibly something that could be captured in a Best System without making it a law (viz., it wouldn't be too costly in terms of simplicity to include an axiom listing all of the sitters and all of the attendees, from which we could derive the needed result as a consequence).

\subsection{Immanent comparisons}

Despite its attractions, MRL faces an important problem in its reliance on intersystem comparisons of simplicity, strength, and balance. The worry as it applies to simplicity has been noted by several authors (Lewis 1983, pp. 366-368, 1986, pp. 123-124; Earman 1986; van Fraassen 1989, pp. 41-43, 51-55; Taylor 1993, p. 82; Loewer 1996, p. 109): it is that assessments of simplicity are relative to an inventory of basic kinds or basic predicates. Adapting terminology from Quine (1970), we can express this point by saying that simplicity is an immanent (defined relative to a system of basic kinds or basic predicates) rather than transcendent (defined independently of the system of basic kinds or basic predicates) notion.

As explained, the MRL account of lawhood demands that we engage in intersystem comparisons of relative simplicity. Unfortunately, however, the immanence of simplicity assessments undercuts the possibility of engaging in such comparisons; for, while we can indeed weigh off the relative simplicity of deductive systems that have a common set of basic kinds (in the formal mode: that use a common set of basic kind predicates), there is no way of making such relative assessments between systems that disagree with respect to the basic kinds (systems that differ in respect of the terms they make available for basic kinds). Loewer (1996) brings out this problem by reference to a famous example from Goodman (1954):

There is a problem concerning the languages in which the best systems are formulated. Simplicity, being partly syntactical, is sensitive to the language in which a theory is formulated, and so different choices of simple predicates can lead to different verdicts concerning simplicity. A language that contains 'grue' and 'bleen' as simple predicates but not 'green' will count 'All emeralds are green' as more complex than will a language that contains 'green' as a simple predicate (p. 109).

Footnote 4 continued

scientific practice. For instance, we might consider that a system merits the simplicity score it gets based on how well it embodies various theoretical virtues, e.g., unification, consilience. Because understanding simplicity this way won't change any of the issues in this paper, we'll continue to think of it as above. 
It has been less frequently noted (but not unnoticed) that the strength of a deductive system is, likewise, immanent rather than transcendent. It is a commonplace that what can be deduced from a set of axioms depends on the available expressive resources and basic predicates (basic kinds); consequently, if strength is a matter of how much can be deduced from the axioms, we lack a way to compare the relative strength of systems that differ in their expressive resources or basic predicates (kinds). What this shows is that, as in the case of simplicity, we lack a means for assessing the relative strength of theories that differ in their expressive resources or basic predicates (kinds).

Finally, the notion of balance is immanent as well. Balance may seem transcendent, for a particular balance metric - e.g., the metric according to which simplicity is twice as important as strength — need not mention any particular set of basic kinds (basic kind predicates). However, to actually obtain a balance score for any particular system, balance requires particular understandings of simplicity and strength and hence inherits the immanence of simplicity and strength. Furthermore, the particular balance metric chosen for a system plausibly hangs on one's notion of explanation. Newtonians who didn't feel a need to posit a mechanism for the gravitational force may have valued simplicity more than critics of Newton's nonlocal force. Inasmuch as explanation is tied to natural kinds, we expect the balance metric to be similarly connected to one's choice of kinds.

Prima facie, the realization that simplicity, strength, and balance are immanent rather than transcendent-what we'll call the problem of immanent comparisons - is a devastating blow to the MRL view. For what counts as a law according to that view depends on what is a Best System; but the immanence of simplicity and strength undercut the possibility of intersystem comparisons, and therefore the very idea of something's being a Best System.

Another way to approach the problem is to consider a trivialization worry raised by Lewis (1983, p. 42, cf. Loewer 1996, p. 109). This difficulty arises by imagining one can compare simplicity, strength and balance while opening up the competition to all kinds. Consider the predicate $\ulcorner F\urcorner$ that holds of all and only things in the world where an arbitrary system $S$ holds. If allowed to compete, it's hard to see why the trivial generalization $\ulcorner(x) F x\urcorner$ wouldn't be the Best System for all such worlds: it is very simple, and also very strong (it strictly implies all truths). ${ }^{5}$ (Strictly speaking, however, we believe the problem of immanent comparisons is prior to this one, for this worry assumes we have (intuitive) transcendent measures that allow $\ulcorner(x) F x\urcorner$ to win in the first place.)

Now, one possible reaction would be to dismiss the problem of immanent comparisons as a product of overheated philosophical imagination. One might hold that all reasonable and realistic standards of simplicity, strength, and balance do converge in their selections of laws about the actual world (even if not in their selections of laws about philosophers' fanciful invented worlds). In something like this spirit, Earman challenges critics to come up with realistic rather than fanciful examples of non-convergence in the MRL laws selected by different best systems:

\footnotetext{
${ }_{5}$ Another way to see the strength of $\ulcorner(x) F x\urcorner$ : if, as per Lewis, individuals are world-bound, then the generalization is maximally informative-it singles out a unique possible world.
} 
I take David Lewis to be saying that in our current state of knowledge we have reason to hope that such cases [viz., cases of non-convergence] do not in fact arise in the actual world. And I take actual scientific practice to be a practical expression of this hope.... I don't have answers to these questions; but I do have a modest suggestion: let us continue the discussion in terms of some concrete examples. Failure to produce them would support Lewis' hope (Earman 1993, p. 418, cf. Loewer 2006, p. 15).

We agree with (what we take to be) the intuition here that not just any imaginable measure of a theory will count as assessing its simplicity or strength, and that not just any deductive system counts as a Best Systematization of the actual facts. However, we don't see that this helps much with the problems under consideration, and regard hopes to the contrary as a form of denial. ${ }^{6}$

Our first complaint about the strategy of denial is that the force of the standard fanciful threats of divergence in simplicity, strength, and balance would survive their non-actuality. It is true that no actual scientists would use a primitive vocabulary on which 'all emeralds are grue' is simpler than 'all emeralds are green'. But this widespread actual convergence does not show that the world forces this choice on us and any would-be Best System. It could have arisen from any number of other sources; most plausibly among these, some have argued that the actual convergence in standards of simplicity underwriting this tendency is the result of an innate but contingent ordering of inductive hypotheses in the human psychological endowment (Fodor 1980). If so, then contingencies of our psychology, rather than any fact about the world, make the envisioned non-convergent standards absent from extant theories. One of the lessons of Goodman's example is that we lack a principled justification for favoring one simplicity metric (hence, set of MRL laws) over another; the fact of widespread actual convergence on one of these does nothing to provide such a justification (contrary to what is suggested by the strategy of denial).

A second reason for concern about denial is that certain historical cases of underdetermination of theory by evidence might reasonably be thought of as revealing the kind of actual non-convergence that denial denies. For example, given the same evidence in the early 1970s, there were over twenty quark models posited in high energy physics - each, no doubt, simpler than others in the eyes of some nonfanciful, sober-minded scientists. Looking to more recent controversies, some debates over the interpretation of quantum mechanics may reasonably be viewed as cases of in principle underdetermination of theory by data, where one theory may be chosen with one conception of simplicity in mind and another by another (equally reasonable and non-fanciful) conception. Such actual episodes in scientific history are prima facie obstacles to the strategy of denial, for they seem to show that there remain a plurality of axiomatizations even after philosophically-inspired fantasies are put to one side. ${ }^{7}$

\footnotetext{
${ }^{6}$ If the suggestion is, by contrast, that the threatening cases won't arise (hence can be safely denied) after we solve the problem of immanent comparisons by some other means, then-depending on the proffered solution-we may agree (more on this later).

7 An MRL proponent bent on denial might respond that, in each of these cases, there really is one preferred, true theory (set of MRL laws) that will be uncovered by the march of scientific progress in the long run. Thus, one might claim that in the long run we'll see a test between rival interpretations of
} 
A final reason for concern about denial is that, unless things go unreasonably well, it will remain unresponsive to the problem of immanent comparisons it was concerned to answer. Recall that that problem arose because, given a diversity of possible axiomatizations of the facts, we lack the transcendent measures of strength, simplicity, and balance that MRL seems to require to arrive at laws. Now, suppose we follow the strategy of denial and reject (through whatever means) the vast majority of possible axiomatizations because they are too far from scientific practice. We still haven't made the problem of immanent comparisons go away, since what we are left with is a plurality of immanent measures of strength, simplicity, and balance (perhaps even one per remaining axiomatization) and zero of the transcendent measures we needed to solve the problem. Put differently, each immanent measure has a Best System associated with it, but there is no transcendent measure available to pick the winner of all of these Best Systems. Of course, if denial could make all but one of the candidates disappear, then we could use its own immanent measures as transcendent measures (since these would apply to all candidate theories - namely, just the one from which they are drawn). But as we have noted, it seems unreasonable to assume that denial will make sense, or that it will whittle the competitors down to a single candidate when it does.

Lewis (1986, p. 124) says that when two or more systems tie for Best Systemhood, the laws are the regularities common to all the "tied" systems and if there is nothing in common then the laws are indeterminate. Perhaps, then, the advocate of denial really hopes that all the resulting non-gruesome systems are effectively tied and endorses the use of the tie-rule. If this modification to MRL is what the denier has in mind, then our point remains: there is no way to tell whether systems are tied, effectively or exactly, without a transcendent measure. And if instead the denier wants directly to search for those generalizations many systems have in common-without the notion of tie-then we consider such a search too free-wheeling to even have any intuitions about.

The problem of immanent comparisons is not that of selecting one from among a range of otherwise acceptable but immanent metrics to apply to a range of axiomatic systems - it is not a problem of choosing one from too many. What is needed to solve the problem is a transcendent simplicity/strength/balance comparison of each axiomatization against others. The problem is not that there are too many immanent measures and nothing to choose between them, but that there are too few (viz., no) transcendent measures.

The problem of immanent comparisons is a serious threat to MRL theories of laws, and the strategy of denial does nothing to eliminate that threat. Consequently, we take it as a constraint on an adequate formulation of MRL that it avoid the transcendent comparisons it can't have, or otherwise resolve the problem of immanent comparisons.

Footnote 7 continued

quantum mechanics or proofs that they are mere syntactic variants of one another, or the dominance of one conception of simplicity over others. But we see no reason for assuming that this must be so in every case, and we certainly don't see that scientific practice conforms to the expectation that it must be so in every case. Consequently, these appeals to the long run of scientific progress threaten to cut MRL off from scientific practice. For this reason, we find that the strategy of denial is seriously undermotivated. 


\subsection{Epistemic accessibility}

Another consideration that we think imposes serious constraints on an adequate MRL concerns epistemic accessibility. We can bring out the issue by comparing MRL against one of its principal Governing View rivals due to Armstrong (1983). Armstrong explains why the pattern expressed by $\ulcorner(x)(F x \supset G x)\urcorner$ holds by positing a necessitation relation $\mathrm{N}(\mathrm{F}, \mathrm{G})$ between universals $\mathrm{F}$ and $\mathrm{G}$. Of course, many questions persist over whether $\mathrm{N}(\mathrm{F}, \mathrm{G})$ really explains the pattern or even entails the pattern; and some ask whether we really have a better handle on $\mathrm{N}(\mathrm{F}, \mathrm{G})$ than we do laws. By far the major complaint, however, is:

$\mathrm{N}(\mathrm{F}, \mathrm{G}) \ldots$ is contingent on another category of facts which transcend the occurrent.... How then do we have epistemological access to N(F,G) (Earman 1986, p. 104)?

Even an ideal observer who sees everything that can be seen in the whole history of the universe cannot be entirely confident to have gotten the laws right (Maudlin 2007, p. 15).

To make this sharp, imagine two possible worlds in which $\ulcorner(x)(F x \supset G x)\urcorner$ holds; in one of which $\mathrm{N}(\mathrm{F}, \mathrm{G})$ is the case but in the other it is not. How, Earman and others ask, could we possibly know which world we're in? If what we observe is the worldly pattern and not N(F,G), we won't know which world we're in. The failure of $\mathrm{N}(\mathrm{F}, \mathrm{G})$ to supervene upon the worldly pattern makes for a radical disconnection between science and the laws.

Another way to put this point is that an adequate MRL should pass Earman's "empiricist loyalty test" (Earman 1986, p. 85). To pass the test, it must be the case that for any two worlds W1 and W2, if W1 and W2 agree on all occurrent facts, then W1 and W2 agree on the laws. Now, as Earman points out, there are many ways of filling in the notion of occurrence, ranging from very strict empiricist (e.g., occurrent is actually observed) through less strict empiricist (e.g., occurrent is observable in principle), through much less empiricist (e.g., occurrent allows unobservable entities like quarks reached through expansive methods such as Glymourian bootstrapping or "inference to the best explanation"). Still, the loyalty test will have bite so long as, for whatever properties one uses to distinguish W1 from W2, we require that there is an epistemological story that gives us warranted epistemic access to the instantiations (non-instantiations) of those properties. The heart of the empiricist loyalty test is just that the properties that distinguish the world where a generalization counts as a law from one where it doesn't must be epistemically accessible.

Even stated this schematically, we can see that the constraint we are now considering raises problems for a theory that uses features such as $N(F, G)$ to distinguish W1 from W2. After all, such features are, virtually by definition, not detectable by standard scientific inquiry. ${ }^{8}$

\footnotetext{
${ }^{8}$ Armstrong et al. will complain, in response, that necessitation relations among universals are preconditions of confirmation and explanation. However, we follow Loewer (1996) in finding this response question-begging (cf. van Fraassen 1989, p. 128).
} 
We take ourselves not to be alone in being attracted to MRL partly because it holds out the promise of a theory of laws that is not radically disconnected from science. Like others, we think that one of the main advantages MRL has over its rivals is that it makes lawhood epistemically accessible in this way, and therefore want to insist that an adequate MRL should preserve that feature.

\subsection{Supervenient kind laws}

The final consideration we'd like to raise in the hope of constraining MRLs involves supervenient kinds. It is beyond controversy that scientists sometimes pose laws in terms of kinds that cannot be understood as the fundamental natural kinds and that couldn't be laws when written in terms of the fundamental natural kinds. This fact is general; it applies to all the special sciences. For a particularly clean example, pretend that fundamental physics has discovered the fundamental natural properties (say, charge, spin, etc.) and consider the laws of thermodynamics. These laws are a powerful and simple set of generalizations that cover the behavior of an astounding variety of substances. Arguably, most or all of these generalizations can be recovered from a statistical postulate on the initial distribution of natural properties and a claim called the Past Hypothesis (Albert 2001), which states that the Boltzmann entropy of the universe was extremely low in the early universe. Philosophers and physicists have often dubbed the Past Hypothesis a law of nature. More than this, the Hypothesis does what an MRLer wants laws to do: it is a proposition that, for a very small price paid in simplicity, gets back an extraordinary amount of strength.

This gives us reason to want a theory of lawhood that at least allows that the Past Hypothesis could be a law. But it's not obvious that MRL can allow this; in particular, this demand will be problematic for a version of MRL that restricts itself to the vocabulary of fundamental physical kinds (below we'll see that the problem generalizes to other forms of MRL). For, after all, entropy is not a fundamental property of physical theory. Furthermore, if we translate the claim that entropy was low into a more fundamental microlanguage we end up with a long gruesome mess. The "law" would be the stipulation that the universe began in one microstate out of an infinitely long disjunction of microstates (all those compatible with the low entropy macrostate of the early universe). The translated claim would still be strong, but hardly simple. For this reason, written in the language of the (ex hypothesi) fundamental kinds, the Past Hypothesis cannot be a MRL law.

The same problem will occur for any putative law employing "supervenient" kinds. Suppose you think — not implausibly-that natural selection is a law of nature. The kind life will be hard to understand as a fundamental kind in a Best System given its supervenience on the kinds of chemistry (etc.) and the attending cost to simplicity through redundancy. And if the high level generalization can be "translated" into the microlanguage as a restriction on the initial conditions of the fundamental primitives, the resulting law will be too long and disjunctive to make it into the Best System. ${ }^{9}$

\footnotetext{
9 Some might not see much of a constraint on MRL here, since a Best System might capture the relevant generalizations at the level of the special sciences as corollaries entailed by its laws, even if not as laws
} 
We suggest that, in order to retain the advertised connection to scientific practice (in which supervenient kind laws are ubiquitous), an adequate MRL should allow for the possibility of laws adverting to supervenient kinds. It must be said that, insofar as there remains hope in the strategy of capturing such generalizations as corollaries rather than entailments (see note 9), this desideratum is less powerful than the others discussed. However, as noted, we don't put much stock in that strategy. In any case, it seems fair to insist that an MRL that allows for supervenient kind laws is ipso facto preferable to one that does not.

Our concern in what follows will be how one can formulate a version of MRL that respects the desiderata we have catalogued.

\section{Fundamentalism}

Consider the problem of immanent comparisons first.

When first faced with this problem, a natural thought would be to restrict all the axiomatizations contending for the title of Best System to a common vocabulary of basic predicates (a common inventory of basic kinds), and thereby obviate the need for transcendent comparisons. Of course, this won't be much of an advance if the set of basic kinds is also up for grabs between the theories. But one might follow Lewis (1986) in holding that, as a matter of fact, certain "perfectly natural" kinds are basic and others are not, and that only axiomatizations expressed in terms of these perfectly natural kinds are candidates for being Best Systems. ${ }^{10}$

Call any version of MRL that solves the problem of immanent comparisons by appeal to a set of perfectly natural fundamental properties a fundamentalist form of MRL. As we say, fundamentalism seems a natural way of responding to the problem of immanent comparisons. Fundamentalism also permits a solution to the trivialization threat raised by the odd predicate $\ulcorner F\urcorner$ —one can simply insist that predicates appearing in laws must (unlike $\ulcorner F\urcorner$ ) pick out a natural kind. However, we now want to show, such fundamentalisms fare poorly with respect to epistemic accessibility and supervenient kind laws.

\subsection{Fundamentalist MRL and epistemic accessibility}

We can raise the problem about epistemic accessibility by noting that fundamentalism seems to allow for the following epistemic possibility. Suppose our scientists

\footnotetext{
Footnote 9 continued

per se. For example, the laws in terms of the fundamental physical properties plus supervenience relations and boundary conditions may entail the generalization that entropy nearly always increases, the principle of survival of the fittest, and so on. On the other hand, there is no guarantee that such a reinterpretation is always available, and to the extent that the scientists themselves don't see it this way, the proponent of MRL who hopes to accommodate the relevant generalizations as corollaries is adding to her story about lawhood a layer of metaphysics not found in the practice of science.

${ }^{10}$ Lewis (1986, pp. 63-69) explores, but remains agnostic between, three accounts of what makes it the case that a property is perfectly natural: primitivism about perfect naturalness, a reductive account in terms of universals, and a reductive account in terms of tropes. This issue won't matter in what follows, so we put it aside.
} 
had complete access to all the facts of the universe and formulated what they considered the best system with the most impeccable methods. Through no fault of their own, still it may be that the system that by their lights best systematizes a field is not the Best System. Why? The scientists might have used the wrong choice of fundamental kinds (vocabulary).

To fill this out a bit, consider the case of Murray Gell-Man and others organizing mesons and baryons into octets-now seen as representations of SU(3) symmetryin what became famous as the Eightfold Way. The theory relies on the positing of new fundamental properties, in particular, fractional charge. The Eightfold Way seems to scientists the on balance strongest and simplest systematization of the relevant phenomena. Is it a law (or at least a corollary of a law)? Of course, it might fail to be because further experiments might reveal more phenomena that demand a better system, or because someone keener than Gell-Man might come along and systematize the field even better. Stipulate for the sake of argument, however, that Gell-Man reasoned impeccably and had all the facts available to him; given the kinds he choose, the Eightfold Way really is the best systematization of the relevant facts. Even granting this much, the fundamentalist cannot guarantee that the Eightfold Way is a law (law corollary); for there is nothing to guarantee that fractional charge is one of the properties enshrined as perfectly natural.

As van Fraassen (1989, p. 53) notes, the opposite can happen as well. Suppose scientists do employ the correct perfectly natural kinds in their theory and form the true Best System with respect to these kinds. Enter now a scientist with a radical theoretical innovation: using other basic kinds, she devises a theory that is intuitively far simpler and more informative than any before. With respect to its novel 'incorrect' choice of kinds, this theory is the best system. But it is not a Best System because, when translated (to the extent it can be translated) into the correct language, it is not as simple as when formulated in its own language. "How could we designate this as an evil day for science?" asks van Fraassen.

MRLers now face a crucial decision. Should we allow the above epistemic possibilities or not?

One (attractively modest) attitude might be that we should allow for the possibility that scientists get the kinds wrong. Scientists are fallible, and our account of laws ought to respect this. Suppose Gell-Man had chosen to formulate the Best System with the property being a guinea pig instead of fractional charge. Would we be so tolerant as to allow that the resulting system produce the laws just because he had access to all the information and reasoned well? The defender of this attitude may point out that the scenarios sketched above are simply instances of skepticism. How we deal with them is a question for our epistemology, not our metaphysics of laws. It may further be pointed out that the fundamental kinds, and hence laws, on this picture, are in the same boat as electrons and other theoretical entities. The skeptical scenario envisioned seems no different than one that an instrumentalist might cook up to impugn realism about the theoretical claims of a science. Instrumentalists commonly imagine possibilities wherein a theory is very successful in its predictive capabilities but not true. The Best System that isn't best is just a variation on this possibility raised with respect to lawhood. 
Crucially, however, if we accept such epistemic possibilities, it seems that we are thereby surrendering epistemic accessibility, and so losing the advantage MRL had over Armstrong's view. For consider again two possible worlds, W1 and W2. In W1 the generalization $\ulcorner(x)(F x \supset G x)\urcorner$ is part of the on balance simplest and strongest system, where $\mathrm{F}$ and $\mathrm{G}$ are perfectly natural kinds, and in otherwise identical W2 the same generalization $\ulcorner(x)(F x \supset G x)\urcorner$ is also part of what scientists think is the Best System, yet F and G aren't perfectly natural kinds. How could we tell what world we're in? We can't: fundamentalist MRL fails the empiricist loyalty test. Normally we can't tell whether what we think is the best system given some kinds really is the Best System, but perfect naturalness adds an extra layer of skeptical possibility. Even if we saw all of world history, possessed unlimited computing power, and so on, we still couldn't determine if $\mathrm{F}$ and $\mathrm{G}$ were perfectly natural, and so, whether $\ulcorner(x)(F x \supset G x)\urcorner$ is really a law of nature.

Again, the problem we are raising with perfect naturalness is not that there is anything intrinsically fishy about it; rather, the problem is that it's hard to imagine a plausible epistemological story that would place naturalness on the right ("occurrent") side of the epistemological divide. Fundamentalism exacerbates an already unnatural feature of MRL. The Best System approach, by its very nature, introduces a metaphysical asymmetry where we have an epistemological symmetry. That is, scientists actually devise laws based on their choice of kinds and choose their kinds based on the laws. Gell-Man was not simply handed fractional charge and left to make the best system he could with it; rather he postulated fractional charge in part because he saw that he could make a very simple and strong system with it if he did. This disconnection between methodology and metaphysics is perhaps tolerable, but when undetectable perfect naturalness is added to the mix, it renders the resulting theory intolerable for any theory of laws that wants to secure epistemic accessibility.

The fundamentalist might attempt to respond by adapting an argument used to defend scientific realism. Scientific realists produce arguments concluding that we have knowledge of unobservable theoretical entities; perhaps $\mathrm{N}(\mathrm{F}, \mathrm{G})$ and primitive naturalness can be the beneficiaries of similar arguments. Alas, we're not optimistic. " Consider, for instance, the famous "no miracles" argument for realism that infers from the remarkable success of science to its truth. Even if one accepts this inference, this doesn't give the fundamentalist what she needs. For it is consistent with the correctness of the inference that the generalization $\ulcorner(x)(F x \supset$ $G x)\urcorner$ is successful and true in both possible worlds considered, but a law in only one of them. The argument must be not only that success and truth are correlated (as per the standard no-miracles argument) but that success and perfect naturalness are correlated. We don't see any reason to believe this is so.

Another possible response for the fundamentalist would involve the idea that the fundamental kinds play the role of not only figuring in laws, but also of acting as "reference magnets" - that the fundamental kinds are in some way more eligible than others to be the referents of our predicates (Lewis 1983, 1984; Sider 2001). If something like this were true, then one wouldn't need an epistemological story to

11 For discussion, see Earman (1986, pp. 105-107). 
insure that the generalizations of the scientist's best system are formulated in terms of the perfectly natural kinds rather than gruesome alternatives. We could instead hold that those generalizations advert to the perfectly natural kinds because only those kinds have the reference magnetism necessary to be referents of the predicates that occur in scientific (and other) generalizations.

Alas, we don't find this answer responsive to the concerns we are raising. For one thing, we are skeptical about the very notion of reference magnetism; although we can see why many have held out hope for reference magnets, we do not understand what such magnetism would amount to substantively, and are not convinced that it exists. More importantly, it seems to us that the appeal to reference magnetism in the current context merely relocates the worries about epistemic accessibility. Remember that we were objecting to fundamentalism on the ground that, since perfect naturalness is (no less than Armstrong's necessitation relation) epistemically inaccessible, the fundamentalist's laws will fail the empiricist loyalty test. But if one started out being concerned that perfect naturalness is epistemically inaccessible, one should be just as concerned that whether perfect naturalness and reference magnetism coincide is epistemically inaccessible. If perfect naturalness and reference magnetism do not coincide, then the appeal to reference magnetism won't help the fundamentalist. For, if they do not, then the generalizations of our best systems will be reference magnetically attracted to less than perfectly natural kinds, and so, given fundamentalism, will express non-laws rather than laws. But it's hard to see that anything could justify us in believing in such an exact coincidence. Consequently, even given all the evidence and impeccable reasoning, there will be no way of knowing, given fundamentalism, whether the Eightfold Way is a law (/law corollary): even allowing that, partly by virtue of reference magnetism, "fractional charge" picks out fractional charge, it will remain epistemically inaccessible whether what "fractional charge" picks out is perfectly natural.

We take all this to show that fundamentalism fails to close off the epistemic possibility we have been contemplating, and so doesn't make lawhood epistemically accessible. Since epistemic accessibility is one of the features that attracted many to MRL over the alternatives, this is a significant drawback to fundamentalist versions of the view.

\subsection{Fundamentalist MRL and supervenient kind laws}

Fundamentalism also has problems accommodating supervenient kind laws. As we noted when first describing the issue in Sect. 1.3, an MRL formulated exclusively in terms of the fundamental, perfectly natural kinds won't recognize as laws generalizations adverting to kinds that supervene on the fundamental kinds but are not among them. If, for example, the inventory of fundamental kinds comes from a descendant of the best current fundamental physics, then it looks highly unlikely that entropy or life will be fundamental. If so, then generalizations employing that kind (such as the extremely simple and strong Past Hypothesis) will be redundant with the MRL laws and so not strong, or, if translated into the microlanguage, then long and disjunctive and so not simple. Either way, it looks highly unlikely that such generalizations could be MRL laws for the fundamentalist. 
Likewise for other generalizations appealing to supervenient kinds such as one finds in every special science.

But, as we have said, a version of MRL that precludes supervenient kind generalizations from counting as laws does less than we want from a theory of lawhood.

\section{Stipulation}

We have argued that, although it solves the problem of immanent comparisons, fundamentalism does not make lawhood epistemically accessible, and does not allow for supervenient kind laws. Let us consider, therefore, another possible response by which the MRLer might hope to answer the problem of immanent comparisons. Namely, suppose we stipulate (as the fundamentalist did) a distinguished set of kinds to solve the problem of immanent comparisons. Judgments of simplicity, strength and balance will then be measured immanently with respect to this distinguished choice of kinds. However, unlike fundamentalists, we could make this distinguished set of kinds something that plausibly passes the empiricist loyalty test. ${ }^{12}$ To use our earlier example, suppose the true generalization $\ulcorner(x)(F x \supset G x)\urcorner$ makes it into our Best System. If $\mathrm{F}$ and $\mathrm{G}$ are empirically accessible, and we have stipulated that $F$ and $G$ are the distinguished kinds, then there is no further metaphysical question that we need answered. There are not two worlds that otherwise look alike, such that in one $\ulcorner(x)(F x \supset G x)\urcorner$ is a law and in the other it is not. The kinds $\mathrm{F}$ and $\mathrm{G}$ are fundamental in the sense of stipulated and distinguished, but not in the sense of unanalysable or scientifically undetectable. For example, if we're told that $\mathrm{F}$ and $\mathrm{G}$ are the stipulated kinds, and furthermore that $\mathrm{F}=\operatorname{ravenhood}$ and $\mathrm{G}=$ blackness, then if (improbably) $\ulcorner(x)(F x \supset G x)\urcorner$ makes it into the Best System, there is no further metaphysical worry for the advocate of MRL. An ideal observer of the world couldn't detect perfect naturalness, but she could, presumably, detect ravenhood and blackness. Thus, this stipulative solution looks as if it is well-suited both to solve the problem of immanent comparisons and pass the empiricist loyalty test. Moreover, the stipulationist can rule out Lewis's trivializing predicate-this time because the property it picks out is not among the stipulated, distinguished (and empirically accessible) kinds.

To bring together variations on the theme, it will be useful to think of stipulative MRLs schematically as views according to which comparisons of simplicity, strength, and balance are relative to some stipulated $X$, where $X$ is a set of kinds that simultaneously solves the problem of immanent comparisons and pass the empiricist loyalty test. There are, of course, many choices of $X$, and so many different forms of stipulative MRL.

One such form is suggested in one of the first clear discussions of the problem of immanent comparisons by Earman (1986):

\footnotetext{
12 Putting things this way makes apparent the close relation between fundamentalism and stipulationism: the former view stipulates a distinguished set of kinds as well (viz., those that are perfectly natural), but in a way that fails the empiricist loyalty test.
} 
... possible worlds can be characterized in terms of space-time magnitudes. Worlds are thus isomorphic to sets of basic propositions, each asserting that the value of such-and-such a magnitude takes a value of so-and-so at thus-andsuch a spatiotemporal location. The laws of the actual world are then the propositions that appear in each of the deductively closed systems of general propositions that achieve a best systemization of the basic propositions true of the actual world. So while different systems may employ different concepts, there will of necessity be a strong common core (p. 90).

For this stipulative MRL, which we might call magnitude $M R L, X$ is a common vocabulary of "relevant physical properties" (13) or "magnitudes" (90) in terms of which distinct axiomatizations can be expressed.

A different form of stipulative MRL-call it macrovariable $M R L$-results from letting $X$ be the macrostates (viz., values of macrovariables such as volume, pressure, temperature, red, blue, etc.) of individuals. Thus, Loewer writes,

A proposal that I think captures what many have on their minds when they speak of fundamental physical properties is that they are the properties expressed by simple predicates of the true comprehensive fundamental physical theory. The true comprehensive fundamental physical theory is the minimal theory that accounts for changes of the locations and motions of macroscopic spatio-temporal entities and also for changes in properties that account for locations and motions and so on (Loewer 1996, p. 110, cf. Loewer 2006, p. 18).

On this view, the Best System is the on balance strongest (i.e., most informative about volume, pressure, color) and simplest (i.e., simplest with respect to the volume, pressure, color carving) systematization of events described by these macrovariables. ${ }^{13}$

Perhaps the limit version of stipulative MRL is the view that identifies $X$ with the observed-we'll call this view observational MRL. The laws of nature on observational MRL are the axioms of the Best Systematization of what is observed. Such a position recalls Poincaré's famous view that the laws of nature were the most convenient assembly of the observations. Many were possible, he thought, and the choice among them conventional. ${ }^{14}$ Each of the stipulative MRLs discussed has important attractions that we pass over here. Suffice to say, arguably each solves the

\footnotetext{
${ }^{13}$ One might worry that the resulting laws would look nothing like ours, that no microscopic kinds and laws would be introduced. We don't see this happening. Just as vacuous laws can make it into the ordinary Best System, so too, laws and kinds about a microscopic world may be needed to get more of the macroworld back. Atoms might be posited to get Brownian motion explained, and so on. Indeed, isn't this the way science works? Scientists don't posit idle kinds and laws at the microlevel. The microworld is warranted if it can ultimately explain and or simplify our description of the phenomena. The debate over atomism at the turn of the last century persisted until tight connections were made between the hypothetical microworld of atoms and the phenomena.

${ }^{14}$ Although commentators on Poincaré often stress the word 'convenience', in fact Poincaré felt that there were hard constraints on our choices - that it might not even be possible for beings like us to choose differently. If we read 'convenience' as maximizing balance, strength, and simplicity, and assume there is a fact about what system is most convenient (and there is some textual evidence for this) then we can read Poincaré as a proponent of stipulative observational MRL.
} 
problem of immanent comparisons and also passes the empiricist loyalty test. As such, each is an improvement over fundamentalism.

\subsection{Once-and-for-all stipulation}

The previous section leaves us with a menu of possible MRL theories, one corresponding to each choice of distinguished kinds $X$. At this point it might seem natural to begin mounting a campaign in favor of some particular set of kinds as special.

We won't do so. While we agree that a theory of laws that is still recognizably MRL-like will always make the laws relative to some choice of kinds, we are against making a single, once-and-for-all choice of kinds that would have the result that would-be best systems formulated in terms of other kinds fail entirely to answer to our concept of law.

We have several concerns about this kind of once-and-for-all stipulationism. We wonder what considerations could confer such privilege on any $X$. The history of science certainly doesn't inspire much confidence that there is a distinguished $X$. Apart from that, we have four objections against building any particular choice of $X$ into our notion of law. The first is that we think the concept of lawhood is plausibly agnostic between particular choices of $X$. Second, as a result, we worry that a onceand-for-all-choice will always be to some extent arbitrary, even after epistemic considerations narrow down the choices of $X$. Third, such a choice doesn't fit well with the MRLer's naturalist-friendly motivations. And, fourth, a single once-andfor-all-choice won't help us recover lawlike generalizations in the special sciences. Let's take these points in order, beginning with the conceptual point.

Why think our concept of lawhood should be independent of particular choices of kinds? Partly because historical disputes between theorists favoring very different choices of kinds seem to us to be disputes between two different sets of laws-not between the recognition of laws as opposed to shmaws. At one point in time classical mechanics was judged to be the best system; now many propose superstring theory as a putative best system. The former uses point-particles as its basic kinds, the latter one-dimensional extended objects. If either were really the Best System, its generalizations would be worthy of being dubbed laws. We claim that our concept of lawhood accommodates classical and stringy kinds. No additional test on the choice of $X$ is required; and this suggests that lawhood tracks being a Best System, not being a Best System formulated in terms of some particular X. Admittedly, it has happened in the history of science that people have objected to particular carvings-most famously, consider the outrage inspired by Newton's category of gravity. But given the link between laws and kinds, this outrage is probably best seen as an expression of the view that another System is Best, one without the offending category. If that other system doesn't in fact fare so well in the best system competition-as in the case of the systems proposed by Newton's foes - then the predictive strength and explanatory power of a putative Best System typically will win people over to the categorization employed. While it's true that some choices of $X$ may strike us as odd, no one would accuse sciencethe enterprise that gives us entropy, dark energy, and charm-as conforming to 
pre-theoretic intuitions about the natural kinds of the world. Yet these odd kinds are all embedded in systematizations that would produce what we would consider laws.

Such considerations convince us that the proposals mentioned in the previous section make demands that our notion of lawhood does not. Magnitude MRL doesn't spell out what the precise magnitudes are, so it scarcely demands any specific choice of $X$. Macroscopic and observable MRL perhaps have better claim on the notion of lawhood. Could systems using different categorizations produce generalizations that qualify as laws? Macrovariable MRL relies on a privileged set of kinds (the macrovariables). We aren't told exactly what variables, but some set of variables, e.g., volume, pressure, macroscopic position, heat, is dubbed special. Observational MRL relies on a privileged set too, those events that are observable. Of course, spelling this theory out in any sort of detail will run into many famous problems. But let's suppose we know what we mean when we say something is observable or macroscopic. Then the question is whether systems that systematize something else can produce what we might call laws. The answer, it seems to us, is yes. When the macrovariable MRLer and the magnitude MRLer disagree, for instance, they are disagreeing about what the laws are-not about whether we should build a science out of laws or a science out of shmaws. ${ }^{15,16}$

Our second worry about making a single once-and-for-all choice of $X$ is that an awful lot hangs on the choice, yet the choice seems somewhat arbitrary. To see this point, consider once again some of the particular $X$ 's mentioned in the previous section.

Begin by considering magnitude MRL. What are the relevant physical properties or magnitudes? Charm, charge, mass, grue, witches? These questions are important to the form of the resulting MRL, since different choices would plausibly result in different theories. For example, consider the effect of the spacetime (set of spacetimes) the theory employs. Aristotelian spacetime uses a notion of absolute rest and absolute velocity; Galilean spacetime does not. Given the tight connection between dynamical and spacetime symmetries, the choice of magnitudes more or less chooses the resulting spacetime. Suppose we stipulate that three-dimensional positions and their velocities on Euclidean space are the common denominator of all systems; we then rule out a priori relativity as a possible set of laws of the Best System. Suppose instead, and more liberally, that we require nothing more than that the fundamental quantities be instantiated on a background spacetime by a manifold; we then rule out a priori various quantum gravitational theories that employ a discrete spacetime lattice rather than a manifold. Suppose we opt for merely a fundamental magnitude instantiated on a four-dimensional structure; we

\footnotetext{
15 Another way to make this point is to note that, since Lewis's perfectly natural fundamental properties are (presumably) non-observable, the systems from which he proposes to build laws are systematizing something other than the observables or the macrovariables. (Note that such a systematization may well still get the observables right.) Yet of all the criticisms of Lewis's theory, no one suggested that its reliance on unobservable kinds renders it a theory of non-laws.

16 If Earman and Roberts (2005) are correct, then to recover the difference between laws and boundary conditions, one ought to restrict the Humean basis to the outputs of reliable measurements; doing so, and then adopting MRL, one would have reason to use these observable outputs as that which gets systematized. We don't want to deny that some choices of $X$ have virtues others don't. We simply don't think any are essential to the concept of lawhood.
} 
thereby rule out superstring theory. All of these prohibitions strike us as potentially arbitrary.

Even choices of $X$ that advert to the observable level can raise these worries. Consider a macrovariable MRLer who picks a select set of macrovariables as special. Of course, another group of scientists might carve the world into macrostates differently. Logically speaking, it's possible that Martian scientists find law/kind systematizations congenial when formulated in the vocabulary of grue and bleen instead of our green and blue. Less fancifully, our own scientists have axiomatized phenomenological thermodynamics in a variety of ways, some using (e.g.) heat as a category and others not. The laws in each system can differ as a result. Is one axiomatization right and the others wrong because heat is or is not in that distinguished set? The history of science has also witnessed a multitude of carvings. Sticking with the thermodynamics example, consider the macrovariable temperature and its changing meanings and extensions. Originally identified with felt hotness, it then was identified with empirical temperature, absolute temperature and now statistical mechanical temperature. In each case a change in carving accompanied a change in laws. What macrovariables we choose to systematize with appears remarkably pliable. Some, like the entropy, the Gibbs free energy, and more, are even somewhat gruesome.

The same threat arises for observational MRL, although the form in which it arises will depend on one's philosophy of perception. Suppose first that observational knowledge is always affected by theoretical assumptions and prejudices (Hanson 1958; Goodman 1976; Kuhn 1970; Churchland 1988). If so-if medieval thinkers simply saw witches (Churchland 1988) — then it is clear that alternative carvings of the observable will lead to different scientific theories, and that a priori, nonprovisional stipulative decisions in favor of one amount to a priori rulings-out of live empirical possibilities. By contrast, suppose that Fodor (1984) is right that the theoretical assumptions in early perception are endogenously fixed in us and "informationally encapsulated" from the influence of higher-level theoretical beliefs. Perception is theoretically biased is particular ways, e.g., assumptions of common objects boundaries or the three-dimensionality of perceived space, but these assumptions are not high-level theoretical ones, and moreover, are shared among everyone. If so, the perceptually available carvings of nature will be sharply curtailed. In fact, however, stipulations have been made, only here by the nature of our perceptual systems rather than our conscious choices. And while it is entirely natural for us to side with the carvings of nature handed to us by our perceptual systems (what other perceptual systems should we follow?), we should not forget that such an policy rules out live possible ways the world might be because of their perceptual inaccessibility. From the point of view of the worry about arbitrariness, MRL laws produced by unchangeable theoretically prejudiced categories resulting from our perceptual apparatus are just as susceptible to the danger as MRL laws produced by changeable theoretically prejudiced categories resulting from our choices.

These considerations suggest that stipulative MRLs may always be objectionably arbitrary.

Third, and related to the second point, a once-and-for-all stipulationism offends against the anti-apriorism that inspires this theory of laws. As we just witnessed, any 
particular choice of $X$ will preclude (what should be) live empirical possibilities. When we choose an $X$ as our stipulated kind, we thereby remove $X$ from the normal back-and-forth of scientific bartering. This result is especially troublesome for MRLers because it is part of their aim to avoid placing constraints on the shape of acceptable scientific theory not justified by broadly empirical inquiry.

Our final worry about a theory of lawhood that rests on a single once-and-for-all choice of kinds is that such a theory will be unable to recover supervenient kind laws using only the stipulated physical kinds. One might try to replace the supervenient kind term in a generalization with its list of subvening basis properties, thereby obtaining a translation of equal strength stated exclusively in terms of the subvening kinds. Granting, arguendo, this can be done, such translations would be long and gruesome (i.e., non-simple) relative to the subvening kinds, and so poor candidates for lawhood in the relevant stipulative MRL. ${ }^{17}$ The upshot is that, so long as we stand by a stipulated standard for (immanent) comparison that excludes supervenient kinds, generalizations stated in terms of those supervenient kinds won't be laws according to the relevant stipulative MRL.

\subsection{Where from here?}

The problems we have raised for stipulative MRL can be seen as resulting from its clinging too tightly to its stipulations. The first cluster of problems is organized around the idea that, in stipulating some fixed background once and for all-kinds, observables, whatever-against which to carry out its simplicity, strength, and balance assessments immanently, stipulative MRLs inappropriately insulate that background from the give and take of empirical inquiry. A further problem is that adherence to any one stipulative basis for immanent comparison renders attractive generalizations expressed in terms of supervening kinds unavailable as MRL laws. The obvious way out of these difficulties, then, would be for the stipulative MRL theorist to treat her stipulations more flexibly.

With respect to the problem about arbitrariness, the proponent of stipulative MRL can treat her stipulated fixed background (of kinds, observables, etc.) as a pro tanto, a posteriori, and defeasible assumption that is not insulated from empirical inquiry. The thought would be that, while some or other stipulated background is needed to carry out the comparisons needed to fix the MRL laws, the background can itself be subjected to rational revision on other occasions. ${ }^{18}$ In slightly more concrete terms, adopting this policy would mean that we could formulate MRL laws for (say) classical physics relative to the background of the kinds of classical

\footnotetext{
17 On the other hand, the contemplated translations won't be available for supervenient kinds not coextensive with any finite disjunction of subvenient kinds. This is plausibly the case for supervenient kinds that are functional, hence realizable in potentially infinitely many different arrangements of the subvenient basis properties (see Fodor 1997).

18 Indeed, just this policy is a standard ingredient of the holistic epistemology often used to describe scientific inquiry; in terms of a familiar image, the lesson is that we cannot repair every plank in Neurath's boat at once, but that every plank in the boat is (even if only in principle) subject to potential repair at some time. It is also a standard ingredient in less holistic epistemologies, such as that of Friedman (2001), according to which the privileged kinds might be viewed as part of what he calls the relativized a priori.
} 
physics, but that we could also make decisions relative to some alternative stipulated basis for comparison about whether the kinds of classical physics should be accepted. (And on yet another day we could reassess that standard for comparison, and so on....) None of the stipulations made at any particular stage would be immune from rational revision, and only pragmatic/explanatory goals (rather than the fixed nature of the universe) would dictate the choice of one so-far empirically supported stipulation rather than another.

The same move could be used to respond to the problem about supervenient kind laws. In particular, if we accept a form of MRL whose comparisons are based on stipulations that change with our explanatory needs, there is no barrier to forming Best Systems, hence laws, relative to the kinds of different stipulated baseswhether those kinds are supervenient or not. (We'll set out this approach to supervenient kind laws in greater detail in Sect. 1.)

\section{Relativization}

Thus, there seems to be a way of understanding stipulative MRL that allows it to evade the worries we have considered; this requires treating the measures of strength, simplicity, and balance in terms of which we understand the notion of a Best System flexibly according to our needs and subject to rational revision. To mark the difference between such a view and the more rigid form of stipulative MRL of Sect. 3, we'll call the new view relativized MRL. This terminology reflects the way in which this view makes Best Systemhood, hence lawhood, relative to a choice among equally available alternatives. ${ }^{19}$ The idea is that even if there is no transcendently Best System (not fixed by nature, not stipulated once and for all by us), there is nothing stopping us from assessing the immanently strongest, simplest, and best balanced axiomatizations relative to a specific choice of basic kinds $K$ (to a specific choice of basic predicates $P_{K}$ ). Given such an assessment relative to a choice of basic kinds $K$ (predicates $P_{K}$ ), we can say that a true generalization is a law relative to $K\left(P_{K}\right)$ just in case it appears in all the immanently Best Systems relative to the basic kinds $K$ (basic predicates $P_{K}$ ). ${ }^{20}$ Of course, since a relativized MRL of this kind makes do without transcendent comparisons, it solves the problem of immanent comparisons. And, so long as the kinds in terms of which it is formulated are epistemically accessible, a relativized MRL will also pass the empiricist loyalty test.

\footnotetext{
19 In fairness, it is not clear that the stipulationists we've cited intend their stipulations in the inflexible way in which we've represented them. Still, it is worth seeing that inflexible stipulations are problematic in order to motivate a more flexible treatment (even if this only clarifies what so-called stipulationists have had in mind all along).

Versions of relativized MRL have been explored and defended by Halpin (2003), Taylor (1993, p. 97), Roberts (1998), Schrenk (2008). Furthermore, it is possible to read Lewis (1983, p. 368) as endorsing a relativized version of MRL in his claim that the laws and kinds must be chosen as a package deal.

${ }^{20}$ Equivalently, if we partition candidate axiomatizations into equivalence classes whose members share sets of basic kinds/predicates, we can say that the laws are relativized to equivalence classes of axiomatizations.
} 
Of course, a version of MRL that relativizes laws to kinds must have something to say about what natural kinds are. An awful lot could be said on this topic; our theory doesn't demand any particular theory of natural kinds. Nevertheless, there exists a very natural answer to this question for proponents of relativized MRL, namely, the popular recent view dubbed 'modest realism' (Kitcher 2003) or 'promiscuous realism' (Dupre 1993, 2001) in philosophy of science, and 'ontological pluralism' or 'explosive realism' in metaphysics (e.g., Carnap 1956; Quine 1969; Taylor 1993; Eklund 2008; Dorr 2008; Sosa 1993; see also the "internal realism" of Putnam 1981).

The answer in question is a proposed middle way between naïve relativism and the idea that nature possesses a uniquely true carving up into kinds; on this view, the world permits possibly infinitely many distinct carvings up into kinds, each equally good from the perspective of nature itself, but differentially congenial and significant to us given the kinds of creatures we are, perceptual apparatus we have, and (potentially variable) matters we care about. Thus some sets of objects, although perfectly objective and well-defined, are not interesting to us, e.g., the set of the Eiffel tower, the two authors, and elm trees, whereas others are, e.g., the set of living creatures.

We say that some such explosive realism about kinds seems a natural mate for relativized MRL because the combination would appear to deliver much of what is wanted by MRL's proponents. Given that relativized MRL depends on some story about kinds, explosive realism seems more attractive than the usual options. ${ }^{21} \mathrm{We}$ take the view to be superior to the traditional naïve conventionalism for which we scold our undergraduates; and it seems more attractive than primitivism or stipulationism about kindhood due to its egalitarianism.

Combining relativized MRL with explosive realism carries further benefits.

For one, it provides an attractive solution to the trivialization threat from Lewis's odd predicate $\ulcorner F\urcorner$ that holds of all and only things in the world where an arbitrary system $S$ holds. If allowed to compete, the generalization $\ulcorner(x) F x\urcorner$ is a Best System for all such worlds since it is very simple, and also very strong. The fundamentalist's and

\footnotetext{
21 There is one respect in which it might seem that explosive realism is particularly less attractive than other options for the proponent of relativized MRL. On the one hand, combining these positions commits one to the view that kinds and laws are relativized to interests, which are presumably intentional states of some kind. But on the other hand, on the usual reductive accounts, intentional states are grounded in reference to kinds and/or laws (see, e.g., Cohen 2004). Therefore, the view seems to be committed to a kind of mutual dependence of interests on laws/kinds and vice versa that might strike some readers as undesirable. We regard this mutual dependence not as a refutation of the position, but instead as a reason for thinking that the position only makes sense if the whole cluster of interests, laws, and kinds comes as a package deal.

It seems to us that much the same can be said about the views defended by Lewis. Recall that, for Lewis, laws, causation, chance, and the rest are all understood in terms of a primitive and interest-relative notion of overall comparative similarity between worlds - which is just to say that, for Lewis, laws, causation, chance, and the rest are relativized to interests (Lewis 1986). But Lewis holds a reductive account of intentional and other mental states, presumably including interests, that is grounded in reference to laws and kinds, inter alia (Lewis 1994b). We take this, too, to be a coherent view, although one whose coherence depends on ultimately taking laws, kinds, and interests (and possibly much more) as a package deal.
} 
stipulationist's response to this worry is to rule out $\ulcorner F\urcorner$ by the requirement that the atomic predicates in competing generalizations must stand for the natural or stipulated properties, respectively. Suppose instead, however, that we accept relativized MRL with explosive realism about kinds. Then we can admit that $\ulcorner(x) F x\urcorner$ is indeed a Best System for our world with all the unfortunate consequences imagined; yet we can remark that $\ulcorner(x) F x\urcorner$ is not a Best System relative to the kinds we care about. If we care about mass, charge, spin, etc., then relative to these kinds, Schrödinger's equation (for example) might result, not 'all events are $F$ '. Properties like $F$ and the ensuing threatened trivialization of MRL are ruled out for lack of interest rather than any intrinsic deficiency.

Another possible benefit is that relativized MRL, like stipulative MRL, can be cast in empiricist form if one wishes: one can relativize the MRL laws to particular sets of observation statements. ${ }^{22}$ Regardless of one's attraction to empiricism in general, we think the availability of an MRL relativized to the observables is important for understanding what happens when scientists posit new fundamental properties. For when entertaining new fundamental properties, they certainly aren't comparing trade-offs relative to the previously recognized physical kinds.

Finally, as suggested earlier, combining MRL with explosive realism will allow for a form of MRL that (unlike many theories of lawhood, including standard MRL) accounts for laws in the special sciences. According to standard MRL, special science generalizations likely will fail to be MRL laws for two reasons. One, as mentioned above, the laws must relate fundamental natural properties, and special science laws won't do so-and if reduced, won't state anything simple. Two, the fact that they tolerate exceptions - the famous "problem of provisos" - and the fact that they are generally incomplete descriptions of reality imply that candidate special science laws will lose in terms of strength to candidate fundamental laws. Arguably, fundamental physics tries ultimately to cover all phenomena; moreover, physical conditions may create exceptions to the special sciences laws.

Some philosophers have concluded from this situation that there are no laws in the special sciences, and instead have tried to explain the special sciences in terms of other (presumably law-independent) apparatus such as causal principles, mechanisms, capacities, powers and dispositions. We find this an over-reaction, and consider it a benefit of relativized MRL that it enables us to accommodate the observations of the anti-law factions without rejecting laws in the special sciences. We cannot do justice to the vast literature on the reasons for doing without laws in the special sciences, but a quick sample of views will isolate the principal motivations.

Woodward (2000) develops a popular alternative non-law-based account of explanation to answer problems that arise in understanding special science generalizations. According to this theory, "whether or not a generalization can be used to explain has to do with whether it is invariant rather than with whether it is lawful. A generalization is invariant if it is stable or robust in the sense that it would

\footnotetext{
$\overline{22}$ Sober (2008) advocates a view of this sort as a way of reconciling empiricism (whose core motivations he wishes to preserve) with the theory-ladenness of observables. According to his theory, one relativizes to sets of observation sentences that presuppose none of the theories under test.
} 
continue to hold under a relevant class of changes" (p. 197). Likewise, Cartwright et al. (2008) propose that special sciences should be explained by what they call "causal principles" rather than laws, the main difference being that causal principles can have exceptions and need not hold everywhere for all time. Once again, the contrast with laws is supposed to lie in a generalization being invariant even when it has exceptions and/or holds over an incomplete range. Finally, although mechanisms and laws are not identical, they are intimately related and the perceived advantages of the former over the latter are often the same as above. Though definitions vary among authors, a mechanism is supposed to be "a complex system which produces that behavior by the interaction of a number of parts according to direct causal laws" (Glennan 1996, p. 52). Later, motivated by Woodward's critique of laws, Glennan (2002), bases the definition on invariances instead; presumably the causal laws invoked are often supposed to be local and with exceptions. Similar claims are made by other mechanists: "In contrast [to laws of nature], models of mechanisms can be highly specific, taking account of the particular factors at work in a specific case in which a phenomenon is studied" (Bechtel and Abrahamsen 2005).

Given this widespread agreement against laws in the special sciences, it is important to stress that there simply are relativized MRL laws in the special sciences, and that furthermore, relativized MRL accommodates the motivations frequently offered by the anti-law crowd. Relativized MRL (when combined with explosive realism about kinds) allows us to axiomatize systems in terms of whatever kinds/predicates we favor; it thereby allows for the construction of Best Systems in botany, ecology, economics, fluid dynamics, high energy particle physics, and plenty more. Every conceivable carving up of the world will, assuming we can make sense of simplicity, strength and balance with respect to these kinds, result in a competition, and where there is a winner, a Best System, hence set of laws. The carvings may be natural-sounding or gruesome-sounding to us. But their availability means that a version of MRL that is relativized to them can allow for laws wherever they are wanted. $^{23}$

Special science MRL laws attractively explain, we think, the sense in which the different sciences are autonomous. The generalizations that become laws are the ones using the immanent standards of the relevant field. Ecologists are not looking over their shoulders at the simplicity, strength and balance metrics of physics. They are using their own metrics tailored to their own field.

What about exceptions and non-universality? Non-universality is no problem at all for relativized MRL. As already noted by Earman (1978, p. 180), even ordinary MRL need not produce laws applicable everywhere and everywhen. A generalization with a highly restricted scope may be sufficiently simpler than any with a less restricted scope, and thereby gain entry into the Best System. This is even more clearly so for relativized MRL. Systems restricted to (say) biological kinds won't have much to say about the universe when it was filled with only light elements, and

\footnotetext{
23 Although not expressed in terms of MRL, Fodor $(1974,1997)$ seems to be after much the same thing in holding that special science laws constrain more worlds (i.e., are stronger) and expressively more compact (i.e., simpler) than the corresponding disjunctive low-level generalizations.
} 
so will be incomplete. Consequently, so long as MRL accounts are viable, the observation that special science generalizations are non-universal is no reason to reject lawhood for the special sciences.

Exceptions require more thought. Exceptions arise because all non-analytic claims will be in principle defeatable by lower-level physical limitations. Consider, for example, Boyle's Law, which says that if the temperature is held constant, the pressure and volume of a gas are inversely proportional to one another. Though a very useful generalization, it has an indefinite number of exceptions. The usual intuition is that such generalizations (including most or all in the special sciences) are legitimate and true despite having such exceptions-or, in the jargon, that they are ceteris paribus (cp) generalizations. The central problem about ceteris paribus generalizations for the philosophy of science is to explain how these generalizations can be both (i) true despite having exceptions, and nonetheless (ii) not vacuous in the way that $\ulcorner p$, unless not- $p\urcorner$ is.

We want to suggest that relativized MRL offers an exciting solution to part but not all of this problem (specifically, it is helpful with respect to (ii) but not (i)). To see this, consider a generalization $\ulcorner p\urcorner$ expressed in terms of the predicates of some special science. Now we can formulate the related generalization $\ulcorner$ ceteris paribus $p\urcorner$. We join a very general consensus in thinking that this second generalization can be true even if (because of the exceptions) the first generalization is not. Unfortunately, we do not have an analysis to offer of the truth conditions for the second generalization, but we take it that almost everyone will accept that it has some, so do not take this as a special burden for our account. ${ }^{24}$ If this is right, then $\ulcorner$ ceteris paribus $p\urcorner$ is something that can be a candidate for inclusion in a Best System. As usual, it will be included iff it is on balance strong and simple relative to the kinds of the relevant field. And we take it that, if $\ulcorner$ ceteris paribus $p\urcorner$ can indeed be true despite having exceptions, then it will make sense to compare it immanently for strength, simplicity, and balance against other true claims expressed in the same vocabulary. (Note that this won't make sense for the unqualified generalization $\ulcorner p\urcorner$.)

This last development is important because it holds out the prospect of a natural and plausible answer to the second half of the outstanding problem of ceteris paribus generalizations: it offers a way of distinguishing the vacuous from the nonvacuous exceptionable generalizations. The thought is that exceptionable generalizations are non-vacuous only if they are part of the Best System for that set of kinds. If Boyle's law, or some law implying Boyle's law, makes it into the Best System for thermodynamic kinds, then that means it is tied to testing, prediction, explanation and all the other facets of a proper scientific enterprise. Cheap

\footnotetext{
24 Just to illustrate that there are live options here, let us quickly indicate a few suggestions that we find congenial. First, one could imagine 'cp' as a kind of theoretical predicate functioning like chance does in Lewis's Best System account. For Lewis, chance is a theoretical term the addition of which gets more generalizations into the Best System. Adding chancy generalizations is a way of getting generalizations that are strong but not always true into a system. One can imagine a similar treatment of 'cp'. Alternatively, one could relax the requirement that the MRL laws be true and replace it with some other requirement, like pragmatic reliability (as determined by the science of interest), much as Lange (2002) does in his account of cp-laws. For other accounts of how cp-generalizations could be true, see Fodor (1989), Pietroski and Rey (1995), Glymour (2002), Schrenk (2008). Again, we won't pick an option here; suffice to say that there are some.
} 
cp-generalizations, by contrast, don't make it in. What makes them cheap is not that they are intrinsically deficient; syntactically and otherwise the cheap cp-generalizations are essentially the same as some perfectly respectable cp-laws. The cheap ones simply fail to play a role in the Best Systematization of the field of interest.

In view of the foregoing, we claim that relativized MRL offers us a way of understanding the special sciences, just as non-special sciences, as involving lawsand this despite the complaints of the opponents of special science laws. To be clear, we do not view relativized MRL laws as a new rival to Cartwrightian capacities, Woodwardian invariances, and so on. Instead, we regard relativized MRL laws as providing some of the missing metaphysics underlying causal principles and invariances. To see this, compare relativized MRL laws with invariances. When a scientist picks a kind/law pair, she implicitly picks out a range of possible worlds wherein some generalization holds. After all, kinds are inherently modal. The set of green things is not merely the set of actual green objects, but the set of actual and possible green objects. So when a scientist considers various laws, or better, law and kind packages, she is implicitly picking out a class of worlds wherein the generalization is stable. This class is precisely the same as Woodward's "relevant class of changes"; for these changes are alterations from one possible world to another. What is important for Woodward is the set of counterfactual interventions/ changes that would or wouldn't alter a given event, for these suggest particular experimental strategies and explanations. But what in the actual world determines the range of possible worlds considered and what happens at these worlds? Woodward doesn't provide an answer, but the answer is clear for relativized MRL: the actual facts systematized by the Best System. If this is right, then not only do the benefits of Woodward's invariances with respect to the special sciences automatically accrue to relativized MRL too, but relativized MRL additionally provides a comparatively clearer account of the underlying metaphysics.

For these reasons, we conclude that relativized MRL, together with explosive realism about kinds, provides a compelling understanding of laws in the special sciences.

\subsection{Relativized MRL, chance, causation, and more}

One of the attractive features of MRL laws-indeed, one of the things that makes these generalizations plausibly laws, and that comes out especially clearly in Lewis's system - is that they are part of a package that includes related notions such as objective chance, counterfactuals, and causation. One might object that a consequence of our relativization is that all the notions that depend on laws of nature are thereby relativized too. Whether one event causes another, whether one counterfactually depends on another, and the chance of an event may all hang on which Best System is employed. And that, it may be thought, is just going too far.

Logically speaking, none of these consequences are forced upon us. We're only offering a theory of laws. Nothing forces us to adopt Lewis-style Humean analyses of causation, counterfactuals, and chances, each of which are tied to the Best System theory of laws. The advocate of relativized MRL could adopt, for instance, a 
hypothetical frequency account of chance; she is not forced to hold a Lewis-style Best System account.

That said, we don't wish to shrink back from the envisioned consequences. Although the relativization of causation, counterfactuals and chances may seem threatening, we're prepared to bite the bullet, explicitly relativize, and explain away intuitions to the contrary by appeal to entrenched interests-just as we have done in accepting laws involving the trivial predicate $\ulcorner F\urcorner$ above. Of course, one need not endorse Lewisian analyses of all these concepts to favor MRL laws; but if MRL laws help with causes, counterfactuals and chances, it would be foolish to dismiss this benefit. Moreover, some extra benefits do follow from this relativization. In particular, as we've seen in the previous section on special science laws, by relativizing we are able to make the metrics of strength, simplicity, and balance sensitive to the concerns of ecologists, biologists, and so on, each of whom may weigh and understand these standards slightly differently. To our minds, this is a great virtue. That this virtue can be spread to causation, counterfactuals, and chance is an additional benefit.

In fairness, this benefit does come with some complexities and potential costs. In particular, one might wonder how we are to understand inconsistency among systems with respect to chance, causation or counterfactual dependence. As an illustration, we'll think this relativization through in the case of chance. Because chance is related directly to rational credence in some ways it presents the hardest case for us. What we say about chance will apply to causation and counterfactuals too, and we'll leave it to the reader to extrapolate our position to those cases.

Suppose, then, that we view chances as the result of a modified Best System competition, where the chances of events are given by the probabilistic laws of the Best System (for details, see Lewis 1994a). If so, then accepting relativized MRL means accepting relativized objective chances, too. This suggests two worries: first, it is not clear what it means for a single event to have different objective chances relative to two different best systems; and second, because objective chances form the basis of rational credence and hence function as part of our guide to life, it is unclear how we should act if different relativized chances guide us inconsistently.

More concretely, suppose we pick out an event $e$ "transcendentally" (say, by pointing)—now the concern is that distinct Best Systems can assign different chances to $e$. The chance of $e$ relative to the Best System restricted to biological kinds is (say) 0.25 . The chance of $e$ relative to the Best System restricted to physical kinds is (say) 0.75 . You are engaged in some important deliberation wherein what you decide hangs on whether $e$ happens. What should you do?

Before responding directly, we'd note that, plausibly, this worry won't arise terribly often for arbitrary Best Systems except when there are reduction relations between them (or one of them is a theory of universal domain), since distinct Best Systems typically operate in their own domains, and since, when there is no reduction relation, these domains are typically non-overlapping. ${ }^{25}$ That said, we

\footnotetext{
25 Moreover, one might try to avoid the worry in cases where it arises by describing $e$ in a more finegrained (immanent) way that would prevent distinct Best Systems from assigning chances to one entity; we'll put this aside for the sake of argument.
} 
think relativized MRL provides the materials for a response to the worry in the cases where it does arise.

As an example where there is arguably reduction (Callender 1999), consider the case of thermodynamics and statistical mechanics. Suppose the event $e$ is the entropy of some closed system decreasing. The Best System relativized to macroscopic thermodynamic kinds, i.e., thermodynamics, is fully deterministic and assigns a chance of 0.00 to $e$. The Best System relativized to statistical mechanical kinds, i.e., statistical mechanics, by contrast, assigns a chance of (say) 0.00000000001 to $e$. However, when we perform the translation we see that one System describes $e$ in more detail than the other. But then, by the so-called Principle of Total Evidence, we ought to base our beliefs and actions on the chance derived from the relativization providing the most information about $e$. (Analogy: when deciding whether to have knee surgery, one would be foolish to base one's decision on the probability of success for male patients if other probabilities are known with respect to a finer class-e.g., chances for those under forty, with a particular surgeon.)

Likewise in the conflict between the chance assignments of classical mechanics and statistical mechanics: if one knows the exact initial position and velocity of the system(s) relevant to $e$, one would be foolish to discard this information. With Hoefer (2007, esp. Sect. 4.4), we stress that both chances in each pair we've considered are perfectly objective, but which one you should use depends on what information one has that bears on the truth of $e^{26}$

What about cases where we have no translation/reduction between the two vocabularies? Here we are stuck; we submit, however, that this is the appropriate answer. If all the information we have about the knee surgery is one doctor saying the chances of success are high and another saying they are low, then we have genuine conflict that can only be resolved when the prior probabilities of the doctors' reliability are taken into account.

In the scientific case, if biology and physics assign conflicting chances to $e$, and there isn't a translation between the vocabularies (or we don't know what it is) that permits us to see one assignment as more informative than the other, then we have a genuine conflict that can only be resolved by consulting the priors. $^{27}$

This answer may seem to conflict with the Principal Principle. The Principal Principle, so named by Lewis, is one type of coordination rule between credences and chances. It says, roughly, that you should set your credence equal to the objective chance no matter what you know about the history of the chance event. In more detail, if $\mathrm{Cr}$ is your credence function, $A$ the proposition of interest, $C(A)$ the chance of $A$, and $E$ further evidence, then the Principal Principle states that:

$$
\operatorname{Cr}(A \mid C(A)=x \& E)=x,
$$

\footnotetext{
${ }^{26}$ Incidentally, a further possible benefit of relativized MRL is that it complements the understanding of chance advocated by Hoefer (2007) in a way that standard MRL does not.

27 In many cases the lower-level theory dominates and is seen as the corrective theory, but there are many cases in the history of science wherein the opposite happens, e.g., geology correcting cosmology over the age of the earth in the nineteenth century.
} 
where $x$ is a number between 0 and 1, inclusive. If we think of the Principal Principle as telling us how to determine our credences, then we don't get a clear rational credence in this case. One can complain that chance isn't a guide to life, as it is sometimes said to be, for we get one rational credence based on the chance relativized to physics, $C_{P}(A)$, and another based on the one relativized to biology, $C_{B}(A)$; only in lucky cases will they both equal $x$. What are we to do?

This complaint is asking the Principal Principle to be more than what it is. The Principal Principle does forge a connection between chances and rational credences; but it is not a general principle of rationality. There is a lot it doesn't tell us about setting our credences. In particular, it doesn't tell us how to set our credences in cases where there are epistemically possible different theories of chance. That is, we can model this case as a situation wherein we are uncertain as to what theory of chance applies to $A$. This situation often arises in science. Two or more viable theories may imply different chances for $A$. We're uncertain as to which theory is correct. In this case our rational credence is determined by summing over all alternative theories of chance, i.e.,

$$
\operatorname{Cr}(A \mid E)=\Sigma_{x} \operatorname{Cr}\left(H_{x} \mid E\right) \cdot \operatorname{Cr}\left(A \mid H_{x} E\right)=\Sigma_{x} \operatorname{Cr}\left(H_{x} \mid E\right) x
$$

where each $H_{i}$ is a hypothesis giving the chance that $A$ is $x$. A theory of chance does not provide the chance that it itself is correct, and it is unreasonable to ask that it should. Supplying this information requires a lot of hard work. With twenty different quark models available in the early 1970s, most giving distinct probabilities to certain events, how should one apportion weight over the different hypotheses? Tough question. But one shouldn't expect one's metaphysics of chance to provide a way out of this problem. Life is hard.

One may object to the foregoing that really there is one Best System for everything. In this case, the uncertainty over chance hypotheses vanishes except for one chance hypothesis. Equation (2) then reduces to equation (1). The advocate of one Best System then doesn't know what to do with the advocate of two Best Systems. We either plug the "correct" chance hypothesis into (1), contra relativization, or we have two instances of (1) with conflicting values for the same event, and no way to reconcile them. We again face conflicting guides about what to do. To import an additional credence function over hypotheses a la (2) seems theoretically inelegant. From this point of view, this additional credence function is doing what the Principal Principle was supposed to be doing, namely, restricting one's priors.

From our perspective, however, this argument gets things backwards. Real life presents us with uncertainties. These uncertainties force us to use something like equation (2). It may be comforting to think that in some ideal metaphysical utopia there is a Single Best System. In that utopia, only one chance hypothesis is epistemically available to us. Then all the headaches involving the use of (2) vanish. But just as the pleasantness of the idea of heaven is no reason to think heaven exists, so the appeal of thinking that one can avoid the difficulties inherent in using (2) is no reason to believe that there is a Single Best System for everything. We have argued that there are many Best Systems, each relative to different sets of kinds and 
interests, and that this view better fits scientific practice than competitor MRL theories do. To grab something hard that is also a part of scientific practice and say that it can be eliminated in a utopian limit is not a desideratum for a theory motivated by scientific practice.

\subsection{Relativized MRL and objectivity}

When they have been considered at all, relativized MRLs have typically been viewed as a position of last resort (e.g., Taylor 1993, p. 97) or worse. The usual reason given for this pessimism concerns the objectivity of the resulting picture.

For example, Earman (1993) worries that making the laws relative to the basic kinds/predicates has the result that "the notion of lawhood would be more subjective than we like to think" (p. 418). Now, assessing this point requires some care. First, note that what the laws of relativized MRL say about the world is just as objective or non-objective as before. If relativized MRL says that, as it might be, the universal gravitational law is a law relative to the basic kinds of classical physics, it has not introduced any subject-dependence into that description of the way masses behave. What is potentially subjective for relativized MRL - at any rate, different from the treatment by other versions of MRL-is not the content of the generalization itself but its status as a law. It is this status, rather than what the generalization says about the world, that relativized MRL makes relative to a choice of basic kinds/predicates.

It is a corollary of this point that relativized MRL does not entail subjectivity in the sense of making scientists (or others) invulnerable to errors about the laws. If relativized MRL makes the generalization 'the color of emeralds is green' a law relative to kinds that include familiar color predicates, then the scientist who rejects that generalization (relative to the same kinds) gets things plain wrong in rejecting a true generalization. Of course, relativized MRL allows that this scientist might get it right about the laws relative to some other set of basic kinds-e.g., one that includes grue and excludes green. But unless her initial aim was to describe the world in terms of these other basic kinds, then that shouldn't give her any comfort.

It is also worth noting that relativized MRL does not make the laws subjective in the sense that they depend for their existence on subjects. This is because the laws (relative to basic kinds $K$ ) that hold of a world $w$ would satisfy relativized MRL's criterion for lawhood-viz., appearing in all the immanently Best Systems for $w$ relative to $K$-whether or not there are subjects in $w$ (or any other world). For relativized MRL, there can be laws (relative to $K$ ) in worlds containing subjects and in worlds not containing subjects.

What is ultimately bothering critics of relativized MRL, we suspect, is just that it delivers immanent rather than transcendent laws. This result sits uncomfortably with the traditional metaphysical aspiration for a theory-neutral characterization of the world independent of us and our conceptual faculties-what Nagel (1986) calls the "view from nowhere" and Williams (1985) calls the "absolute conception of the world." Relativized MRL adds to the reasons to doubt the possibility and intelligibility of such a characterization by offering a picture of laws that is essentially perspectival. (It bears repeating, however, that the perspectives in question need not be subject-dependent.) 
One symptom of this concern is that some philosophers encountering this view worry about whether theory change can ever be rational if relativized MRL is correct. Suppose scientist A is interested in kinds $X_{A}$ and scientist B is interested in kinds $X_{B}$. Both, let us suppose, flawlessly formulate the Best System for each set of kinds, respectively. Is there any reason for scientist A to ever switch to scientist B's system? To induce the intuition that there should be, let's suppose Scientist A is someone immersed in the Ptolemaic system and Scientist B someone immersed in the Copernican system. Here we have a feeling that Scientist A is using the wrong kinds/laws package and should switch. But why should she, the objection goes, when there is no transcendent measure to compare systems? Sure, by Copernicus's time the Ptolemaic system arguably required up to 70 epicycles to maintain empirical adequacy. Probably it didn't score well on the simplicity standard. By not sharing the same kinds, however, the Copernican system can't be compared with the Ptolemaic on simplicity or even, for that matter, strength or balance. (Of course, one can repeat this argument for virtually any case of theory change.)

Obviously this is a large issue, one of the deepest in all of philosophy. As it applies to us, the idea behind the objection is that relativized MRL doesn't have the transcendental structure necessary to make Scientist A irrational if she doesn't switch. The world doesn't dictate a "best of the best" kind and law package. The relativized MRLer, in other words, must be a kind of Carnapian or Kuhnian with respect to theory change, explaining the change from one theory to another as always the result of explanatory/pragmatic needs and not rational compulsion.

Of course, this issue wouldn't be such a large one if matters were so clear-cut. Suffice to say, we largely agree that relativized MRL is consonant with a broadly Carnapian picture of theories (although we disagree that it is Kuhnian, at least according to some common readings of Kuhn), but think that this is hardly reason for dismissal. ${ }^{28}$ In response to the present objection, the Carnapian has a number of responses, most of which try to show that in historical transitions where we feel it rational for one side to change, there really are hidden shared standards, internal reasons for abandoning one theory for another, and so on. The case where no internal reasons at all exist so that rational persuasion can't occur is viewed as a kind of imaginary limit case not corresponding to any actual scientific episode. The relativized MRLer can parrot all the usual moves, pointing out the many immanentfriendly reasons one might want to switch systems, e.g., Ptolemaic cosmology wasn't really so simple or strong, even by its own standards.

The only novel point MRL contributes to these deep controversies, so far as we can see, is the possibility of modeling many of them via its flexibility. Scientists A and B disagree, but they might agree that getting (say) the observables, suitably characterized, is important. They could then formulate the Best System relative to the observables of interest, which would be a third preferred $X, X_{C}$. At this point they can ask: is the on balance strongest and simplest system really one requiring the system of concentric spheres? By framing debates between different kind/law

\footnotetext{
${ }^{28}$ See Friedman (2001) for a contemporary take on theory change from a Carnapian perspective.
} 
pairs in terms of debates over the best systematization of the observables (or some other common body of knowledge), we submit that relativized MRL can model cases of theory change where it seems compelled.

Despite what has been said, we anticipate that some will persist in finding that the immanent character of relativized MRL makes it insufficiently objective. However, for whatever it is worth, we don't find philosophers' discomfort with the immanent character of relativized MRL a decisive objection against the view. Indeed, such discomfort is predictable, given the truth of relativized MRL, on the (plausible) assumption that we often fail to notice how our scientific search for laws is informed by our commitments about kinds except during scientific episodes when the kinds are explicitly contested.

\section{Conclusion}

As stated at the outset, we'd like to defend the MRL theory of lawhood if we can. We think that working through the problems discussed in this paper leads not only to a more nuanced understanding of the possibilities and prospects for a wide range of MRLs, but to an appreciation of what the most promising form of that view will look like.

We have argued that, by adopting an MRL whose laws are relativized to a class of basic kinds/predicates, we can have a theory grounded in immanent rather than transcendent comparisons. Moreover, the resulting view will make lawhood epistemically accessible. We suggest that the kinds/predicates to which MRL laws are relativized must be open to the process of rational inquiry, rather than stipulated once and for all, lest the resulting MRL become both objectionably non-deferential to scientific practice and divorced from its history. Of course, we want to allow (indeed, encourage) diversity in the choice of kinds/predicates to which MRL laws are relativized in order to allow for MRL laws in diverse domains, very much including the domains of the special sciences.

As we see matters, a view of this sort inherits the advantages of other positions while avoiding their defects, and that it can be defended from many of the most important objections it faces. Consequently, we claim that relativized MRL is the best available version of the theory. If we are right in preferring MRL to other theories of laws, then our favored flavor of MRL represents not only the best of Best System theories, but the best theory of lawhood simpliciter.

Acknowledgments We are grateful to Peter Bokulich, Nancy Cartwright, Shamik Dasgupta, Adam Elga, Michael Glanzberg, David Hilbert, Carl Hoefer, Nick Huggett, Colin Klein, Barry Loewer, Ulrich Meyer, Chris Meacham, Jill North, Jonathan Schaffer, Joshua Schechter, Ted Sider, Paul Teller, Brian Weatherson, and an audience at the 2008 Bellingham Summer Philosophy Conference for discussion that has much improved this paper.

Open Access This article is distributed under the terms of the Creative Commons Attribution Noncommercial License which permits any noncommercial use, distribution, and reproduction in any medium, provided the original author(s) and source are credited. 


\section{References}

Albert, D. Z. (2001). Time and chance. Cambridge, MA: Harvard University Press.

Armstrong, D. M. (1983). What is a law of nature. Cambridge: Cambridge University Press.

Ayer, A. J. (1956). What is a law of nature? Revue Internationale de Philosophie, 10, 144-165.

Bechtel, W., \& Abrahamsen, A. (2005). Explanation: A mechanistic alternative. History and Philosophy of the Biological and Biomedical Sciences, 36, 421-441.

Berryman, A. A. (2003). On principles, laws and theory in population ecology. OIKOS, 101(3), 695-701.

Callender, C. (1999). Reducing thermodynamics to statistical mechanics: The case of entropy. The Journal of Philosophy, 96(7), 348-373.

Carnap, R. (1956). Empiricism, semantics, and ontology. In R. Carnap (Ed.), Meaning and necessity: A study in semantics and modal logic (2nd ed., pp. 203-221). Chicago: University of Chicago Press.

Cartwright, N. (1983). How the laws of physics lie. New York: Oxford University Press.

Cartwright, N., Alexandrova, A., Efstathiou, S., Hamilton, A., \& Muntean, I. (2008). Laws. In M. Smith \& F. Jackson (Eds.), Oxford handbook of contemporary philosophy. Oxford: Oxford University Press.

Churchland, P. M. (1988). Perceptual plasticity and theoretical neutrality: A reply to Jerry Fodor. Philosophy of Science, 55(2), 167-187.

Cohen, J. (2004). Information and content. In L. Floridi (Ed.), Blackwell guide to the philosophy of information and computing (pp. 215-227). New York: Basil Blackwell.

Colyvan, M. (2003). Laws of nature and laws of ecology. OIKOS, 101(3), 649-653.

Dorr, C. (2008). What we disagree about when we disagree about ontology. In M. E. Kalderon (Ed.), Fictionalist approaches to metaphysics. Oxford: Oxford University Press.

Dretske, F. (1977). Laws of nature. Philosophy of Science, 44, 248-268.

Dupré, J. (1993). The disorder of things: Metaphysical foundations of the disunity of science. Cambridge, MA: Harvard University Press.

Dupré, J. (2001). Human nature and the limits of science. New York: Oxford University Press.

Earman, J. (1978). The universality of laws. Philosophy of Science, 45, 173-181.

Earman, J. (1986). A primer on determinism. Holland: Dordrecht.

Earman, J. (1993). In defense of laws: Reflections on Bas van Fraassen's laws and symmetry. Philosophy and Phenomenological Research, LIII(2), 413-419.

Earman, J., \& Roberts, J. (2005). Contact with the nomic: A challenge for deniers of humean supervenience about laws of nature part I: Humean supervenience. Philosophy and Phenomenological Research, 71(1), 1-22.

Eklund, M. (2008). The picture of reality as an amorphous lump. In J. Hawthorne, T. Sider, \& D. Zimmerman (Eds.), Contemporary debates in metaphysics. Oxford: Blackwell.

Feynman, R. P., Leighton, R. B., \& Sands, M. (1963). The Feynman lectures on physics. Reading, MA: Addison-Wesley.

Fodor, J. A. (1974). Special sciences. Synthese, 28, 97-115.

Fodor, J. A. (1980). Fixation of belief and concept acquisition. In M. Piatelli-Palmerini (Ed.), Language and learning: The debate between Jean Piaget and Noam Chomsky (pp. 143-149). London: Routledge \& Kegan Paul.

Fodor, J. A. (1984). Observation reconsidered. Philosophy of Science, 51(1), 23-43.

Fodor, J. A. (1989). Making mind matter more. Philosophical Topics, 17, 59-79 (Reprinted in A theory of content and other essays, pp. 137-159, by J. A. Fodor, 1990, Cambridge, MA: MIT Press).

Fodor, J. A. (1997). Special sciences: Still autonomous after all these years (a reply to Jaegwon Kim's "multiple realization and the metaphysics of reduction"). In J. E. Tomberlin (Ed.), Philosophical perspectives, 11: Mind, causation, and world (pp. 149-163). Atascadero, CA: Ridgeview Press.

Friedman, M. (2001). Dynamics of reason. Stanford, CA: CSLI Publications.

Giere, R. (1999). Science without laws. Chicago: University of Chicago Press.

Glennan, S. (1996). Mechanisms and the nature of causation. Erkenntnis, 44, 49-71.

Glennan, S. (2002). Rethinking mechanistic causation. Philosophy of Science, 69, S342-S353.

Glymour, C. (2002). A semantics and methodology for ceteris paribus hypotheses. Erkenntnis, 57, 395-405.

Goodman, N. (1954). Fact, fiction, and forecast. Cambridge, MA: Harvard University Press.

Goodman, N. (1976). Languages of art: An approach to a theory of symbols. Indianapolis: Hackett.

Halpin, J. F. (2003). Scientific law: A perspectival account. Erkenntnis, 58, 137-168. 
Hanson, N. R. (1958). Patterns of discovery: An inquiry into the conceptual foundations of science. Cambridge: Cambridge University Press.

Hoefer, C. (2007). The third way on objective probability: A sceptic's guide to objective chance. Mind, 116(463), 549-596.

Kitcher, P. (2003). Science, truth, and democracy. New York: Oxford University Press.

Kuhn, T. S. (1970). The structure of scientific revolutions (2nd ed.). Chicago: University of Chicago Press.

Lange, M. (2002). Who's afraid of ceteris-paribus laws? or: How I learned to stop worrying and love them. Erkenntnis, 57(3), 407-423.

Lewis, D. (1973). Counterfactuals. Oxford: Basil Blackwell.

Lewis, D. (1983). New work for a theory of universals. Australasian Journal of Philosophy, 61, 343-377.

Lewis, D. (1984). Putnam's paradox. Australasian Journal of Philosophy, 62, 221-236.

Lewis, D. (1986). On the plurality of worlds. Oxford: Basil Blackwell.

Lewis, D. (1994a). Humean supervenience debugged. Mind, 103(412), 473-490.

Lewis, D. (1994b). Reduction of mind. In S. Guttenplan (Ed.), A companion to the philosophy of mind (pp. 412-431). Oxford: Blackwell.

Loewer, B. (1996). Humean supervenience. Philosophical Topics, 24(1), 101-127.

Loewer, B. (2006). Laws and natural properties (unpublished).

Maudlin, T. (2007). The metaphysics within physics. New York: Oxford University Press.

Nagel, T. (1986). The view from nowhere. Oxford: Oxford University Press.

Pietroski, P., \& Rey, G. (1995). When other things aren't equal: Saving ceteris paribus laws from vacuity. British Journal for the Philosophy of Science, 46(1), 81-110.

Putnam, H. (1981). Reason, truth, and history. New York: Cambridge University Press.

Quine, W. V. O. (Ed.) (1969). Natural kinds. In Ontological relativity and other essays (pp. 114-138). New York: Columbia University Press.

Quine, W. V. O. (1970). Philosophy of logic. Cambridge, MA: Harvard University Press.

Roberts, J. (1998). 'Laws of Nature' as an indexical term: A reinterpretation of Lewis's best-system analysis. Philosophy of Science, 66(Supplement), 502-511.

Schrenk, M. (2008). A theory for special sciences laws. In H. Bohse, K. Dreimann, \& S. Walter (Eds.), Selected papers contributed to the sections of GAP.6, 6th international congress of the society for analytical philosophy. Paderborn: Mentis.

Shoemaker, S. (1998). Causal and metaphysical necessity. Pacific Philosophical Quarterly, 79, 59-77.

Sider, T. (2001). Four-dimensionalism: An ontology of persistence and time. Oxford: Clarendon.

Sober, E. (2008). Empiricism. In S. Psillos \& M. Curd (Eds.), The Routledge companion to the philosophy of science. New York: Routledge.

Sosa, E. (1993). Putnam's pragmatic realism. The Journal of Philosophy, XC(12), 605-626.

Swartz, N. (1985). The concept of law. New York: Cambridge University Press.

Swoyer, C. (1982). The nature of natural laws. The Australasian Journal of Philosophy, 60, 202-223.

Taylor, B. (1993). On natural properties in metaphysics. Mind, 102, 81-100 (Reprinted in The Philosopher's Annual, XVI, 185-204, 1993).

Tooley, M. (1977). The nature of law. Canadian Journal of Philosophy, 7, 667-698.

van Fraassen, B. C. (1989). Laws and symmetry. Oxford: Clarendon.

Ward, B. (2002). Humeanism without Humean supervenience: A projectivist account of laws and possibilities. Philosophical Studies, 107, 191-218.

Weinberg, S. (1987). Toward the final laws of physics. In R. Feynman \& S. Weinberg (Eds.), Elementary particles and the laws of physics. Cambridge: Cambridge University Press.

Williams, B. (1985). Ethics and the limits of philosophy. Cambridge, MA: Harvard University Press.

Woodward, J. (2000). Explanation and invariance in the special sciences. British Journal for the Philosophy of Science, 51, 197-254. 